\title{
Emergency medicine in Paarl, South Africa: a cross-sectional descriptive study
}

\author{
Rens Hanewinckel • Henri P. Jongman • Lee A. Wallis • \\ Terrence M. Mulligan
}

Received: 17 November 2009 /Accepted: 19 April 2010/Published online: 9 July 2010

(C) Springer-Verlag London Ltd 2010

\begin{abstract}
Background Emergency Medicine (EM) in South Africa is in its earliest stages of development. There is a paucity of data about emergency department (ED) patient demographics, epidemiology, consultation and admission criteria and other characteristics.

Aims This information is absolutely necessary to properly guide the development of EM and appropriate emergency care systems. In order to provide this information, we performed a study in a rural hospital in Paarl, $60 \mathrm{~km}$ outside Cape Town.

Methods All patients who were seen in the ED between 1 January 2008 and 31 May 2008 were eligible for our research. We designed a cross-sectional descriptive study and retrieved information from a randomized sample of all consecutive patient charts seen during this period using a 40-point questionnaire (see Appendix 1).
\end{abstract}

Disclaimer: The views expressed in this paper are those of the author (s) and not those of the editors, editorial board or publisher.

\section{R. Hanewinckel $\cdot$ H. P. Jongman $(\square)$ \\ Erasmus University, \\ Rotterdam, The Netherlands \\ e-mail: henrijongman@hotmail.com}

\section{A. Wallis}

Emergency Medicine,

University of Cape Town \& Stellenbosch University,

Cape Town, South Africa

\section{T. M. Mulligan}

Emergency Department and Emergency Medicine Residency,

Universitair Medisch Centrum Utrecht,

Utrecht, The Netherlands
Results We investigated 2,446 charts, of which 2,134 were suitable for our research The majority $(88.2 \%)$ of these patients were self-referred. In our sample, $24.1 \%$ were children under 12 years old. Almost $20 \%$ of patients had a serious pathological condition or were physiologically unstable; $36.0 \%$ of all presentations were trauma related. Besides trauma-related problems, gastrointestinal- $(21.9 \%)$ and respiratory tract- $(12.4 \%)$ related problems were most common in the ED; $16.5 \%$ of the patients were admitted to a ward.

Conclusion This descriptive epidemiological study provides necessary data that will be used for further needs assessments and for future EM development in Paarl, and can be used as a template in other EDs and hospitals to provide similar data necessary for initial EM development strategy.

Keywords Emergency medicine $\cdot$ South Africa . Demographics · Violence

\section{Introduction}

In South Africa, the rates of violence and trauma have been reported as some of the highest in the world [1]. Nationally, 2.5 million cases of non-fatal injuries require emergency care, and an estimated 60,000 South Africans die of homicides and road traffic accidents each year: this translates to about 66 trauma presentations per 1,000 population per annum [2]. Diseases of the developing world also remain prominent; epidemiological and demographic shifts (caused by increased urbanization, aging of the population and gradual Westernization of lifestyles) have led to the emergence of noncommunicable diseases, such as heart disease and cancer, as a major problem [1]. These factors combine to create situations 
in which well-developed emergency medicine (EM) and emergency care systems are essential.

In 2004 EM was established as an official new specialty [3-5]. To meet the requirements of the specialty, EM specialists, EM training and a good working emergency care system are necessary. This includes appropriate emergency departments (ED) and emergency medical services (EMS) [5].

Previous estimates of ED workload in Cape Town have demonstrated that a significant proportion of presenting populations are emergency or urgent in nature, present to the ED outside of normal office hours, involve a large paediatric case mix and are typically walk-in or self-referred [6, 7]. There is a paucity of data about ED patient demographics, epidemiology and other characteristics in South Africa in general. This information is necessary to properly guide the development of appropriate emergency care systems. Previous articles have described the workload at level one [6] and urban secondary level hospitals [7], but there are no data on the situation in rural secondary level hospitals.

In order to provide this information, we designed this cross-sectional, descriptive study in a rural secondary level hospital.

\section{Materials and methods}

Study location

Paarl is located in the mountainous wine region outside of Cape Town, South Africa. Paarl Hospital is a 250-bed secondary level facility, which serves the needs of 600,000 people in a vast geographical, rural area; the population served is mainly uninsured.

Study population

We undertook a retrospective study of patients seen in the ED; all patients presenting to the ED between 1 January and 31 May 2008 were eligible for inclusion.

We used a standardized data extraction tool (Appendix 1) for data collection.

Patient folders were selected by random sample (based on the patient's identification number), using a random number generator. The sampled population was all patients who presented during this time.

Charts lacking the ED patient entry, or lacking all essential data points, were excluded.

Statistical analysis

Data were entered into a SPSS Data Entry Station 4.0 (SPSS Inc. 2003) database and were analyzed using SPSS
15.0 (SPSS Inc. 2006) for Windows. $P$ values were calculated using a confidence interval analysis program (CIA version 1.0, 1989).

\section{Results}

A total of 16,996 patients presented during the study period. Of these, 2,134 were fully analyzed (Fig. 1).

Sample characteristics

There were $53.7 \%$ men $[54.1 \%$ in the reference population $(P>0.05)]$ and $512(24.1 \%)$ were children aged $0-12$ years (of whom $68.9 \%$ were under 4 years of age): there was no significant difference in the percentage of children from the reference population $[23.4 \%(P>0.05)]$.

Referral and transportation

Referral information was available for 1,781 patients (83.6\%); of these, most were self-referred $(88.2 \%)$ - other patients were referred from clinics $(6.9 \%)$, general practitioner $(4.1 \%)$ or a specialist $(0.8 \%)$.

Transport information was available in $1,841(86.3 \%)$ of cases: $26.9 \%$ of these arrived by ambulance, $3.7 \%$ by police and the rest self-presented.

Time distribution

Days were split into six 4-h sessions; time data were available for all patients. Overnight periods were relatively quiet; there were no significant differences in the four periods between 0800 and 2400 hours (Fig. 2).

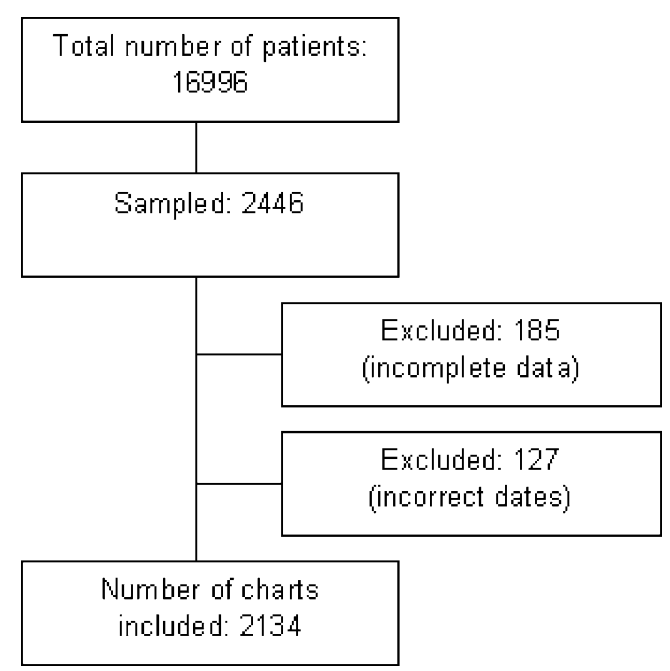

Fig. 1 Sample 


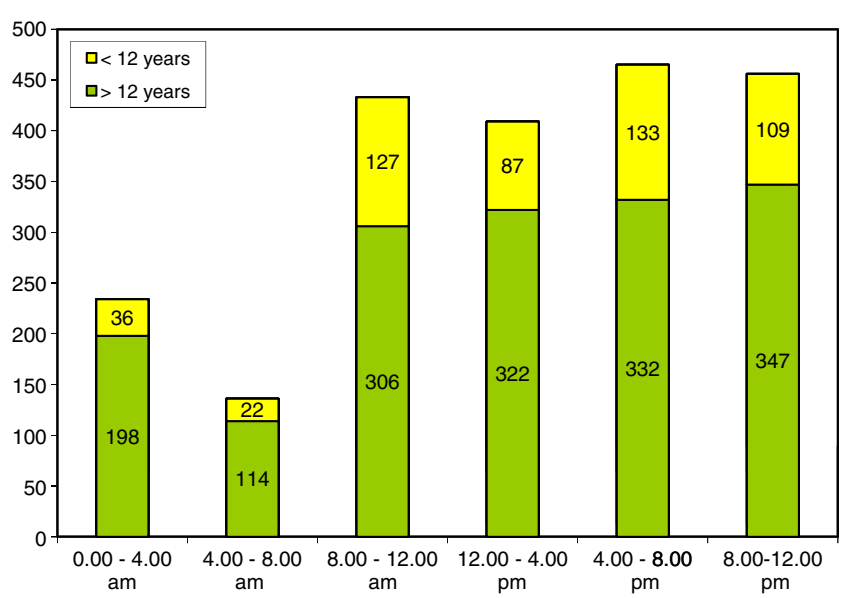

Fig. 2 Temporal arrival of patients $(n=2,134)$

Presentation times of trauma patients followed similar time patterns.

Time from arrival to triage, triage to doctor and doctor to disposal were too sporadically recorded to derive meaningful data.

Triage category

Paarl Hospital triages using the South African Triage Scale (SATS) [8]. This system divides patients into five groups:

- Green: minor injury/illness

- Yellow: physiologically stable cases with reasonably serious medical or trauma problems

- Orange: serious cases, with potentially unstable physiology or potentially life/limb-threatening pathological condition

- Red: resuscitation/physiologically unstable patients

- Blue: clearly dead

Triage information was available in 1,147 cases: overall $4.9 \%$ were red, $14.3 \%$ orange, $66.9 \%$ yellow and $13.9 \%$ green (with 1 blue patient).

There was no significant difference in severity between children and adults. We combined the yellow and green group and compared this with the combined red and orange group. For adults the less serious cases (yellow and green) accounted for $80.5 \%$ and the more serious cases (red and orange) for $19.4 \%$ and for children 81.6 and $18.4 \%$, respectively (both $P>0.05$ ). We made the same analysis for self-presenting patients vs EMS patients. There was no significant difference in triage severity between self-presenting and EMS patients (both $P>0.05$ ). Self-presented patients had a serious pathological condition in $18.6 \%$ and EMS in $20.5 \%$ and less serious complaints in 81.4 and $79.5 \%$, respectively.
Presenting complaint

Trauma was the commonest presenting complaint, occurring in $36 \%$ of cases. The commonest complaints are shown in Table 1 (several patients presented with more than one complaint).

With regard to the trauma patients, $65.7 \%$ were male, $56 \%$ were blunt in nature (predominantly falls or assault with a blunt item), $33.4 \%$ penetrating (although there were no gunshot wounds in our sample), $2.6 \%$ sexual assault and $2.5 \%$ burns (the cause was unknown in 42 cases).

\section{Diagnosis}

A total of 2,503 diagnoses were made in 2,134 patients (Table 2). These often did not correspond with the recorded presenting complaint.

The five commonest diagnostic categories accounted for $70 \%$ of patients: they were further analyzed (Table 3 ).

Treatment

A total of 1,097 (51.4\%) patients had 1,394 procedures undertaken - including suturing, placing IV lines and chest tubes, and applying bandages and plasters.

Medications were administered in $87.1 \%$, with more than half of these receiving analgesia $(1,131,53.0 \%), 593$ $(27.8 \%)$ antibiotics and $5.9 \%$ nebulized medication.

\section{Disposition}

A total of 565 patients $(26.5 \%)$ were observed in the ED or the observation ward. Ward admission occurred in 353 $(16.5 \%) ; 47$ patients were transferred to another facility, and

Table 1 Commonest presenting complaints

\begin{tabular}{ll}
\hline Presenting complaint & $\%$ \\
\hline Trauma related & 36.0 \\
Gastrointestinal tract & 21.9 \\
Respiratory tract & 12.4 \\
Nervous system & 8.3 \\
Musculoskeletal & 7.3 \\
Systemic/metabolic & 6.6 \\
Cardiovascular & 5.1 \\
Genitourinary tract & 4.3 \\
Ear nose throat & 4.3 \\
General weakness & 3.7 \\
Skin & 3.6 \\
Intoxicated & 2.0 \\
Psychiatric & 1.5 \\
Ophthalmic & 1.1 \\
\hline
\end{tabular}


Table 2 Diagnostic category

\begin{tabular}{ll}
\hline Diagnostic category & $n(\%)$ \\
\hline Trauma & $826(33.0)$ \\
Respiratory tract & $372(14.9)$ \\
Gastrointestinal tract & $330(13.2)$ \\
Genitourinary tract & $125(5.0)$ \\
Nervous system & $116(4.6)$ \\
Skin & $108(4.3)$ \\
Ear nose throat & $106(4.2)$ \\
Cardiovascular & $104(4.2)$ \\
Systemic/metabolic & $94(3.8)$ \\
Musculoskeletal & $77(3.1)$ \\
Intoxication & $50(2.0)$ \\
Psychiatric & $21(0.8)$ \\
Ophthalmic & $20(0.8)$ \\
Unknown & $154(6.2)$ \\
\hline
\end{tabular}

13 patients $(0.6 \%)$ died in the ED. A total of $200(9.4 \%)$ did not complete their visit - 146 left without being seen by a doctor (LWBS) and 54 left against medical advice (LAMA).

Disposal by diagnostic category is shown in Table 4 .

\section{Discussion}

There is currently a general paucity of data concerning patient profiles in EDs in South Africa. This is essential background information to guide development of EDs, emergency care systems, and to develop the specialty of EM appropriately. Two prior reports have highlighted patient profiles in district (primary level) [6] and urban regional (secondary level) hospitals [7]. In addition to these prior studies, our study adds essential information by addressing the situation in a rural secondary level hospital.

Table 3 Most common diagnoses

\begin{tabular}{|c|c|c|c|}
\hline Diagnosis & $\%$ & Mean age (years) & $\%$ Age $<12$ years \\
\hline \multicolumn{4}{|l|}{ Trauma } \\
\hline Laceration head & 20.3 & 31 & 10.7 \\
\hline Soft tissue head & 9.0 & 23 & 29.7 \\
\hline Laceration hand & 5.3 & 28 & 9.2 \\
\hline Laceration arm & 5.0 & 29 & 9.8 \\
\hline Laceration back & 4.2 & 32 & 2.9 \\
\hline \multicolumn{4}{|l|}{ Respiratory tract } \\
\hline Pneumonia & 29.8 & 24 & 48.6 \\
\hline URTI & 23.1 & 8 & 86.0 \\
\hline РТВ & 14.8 & 44 & 5.5 \\
\hline Bronchospasm & 8.6 & 41 & 28.1 \\
\hline Asthma & 8.1 & 36 & 30.0 \\
\hline \multicolumn{4}{|l|}{ Gastrointestinal tract } \\
\hline Gastroenteritis & 44.2 & 18 & 63.0 \\
\hline Gastritis & 9.1 & 31 & 10.0 \\
\hline Constipation & 8.5 & 37 & 17.9 \\
\hline Abdominal pain & 6.1 & 36 & 5.0 \\
\hline Dysentery & 3.9 & 20 & 61.5 \\
\hline \multicolumn{4}{|l|}{ Genitourinary tract } \\
\hline Urinary tract infection & 25.6 & 42 & 0 \\
\hline Miscarriage & 12.0 & 24 & 0 \\
\hline Pyelonephritis & 9.6 & 35 & 8.3 \\
\hline Renal failure & 7.2 & 59 & 0 \\
\hline Pregnancy & 5.6 & 26 & 0 \\
\hline \multicolumn{4}{|l|}{ Nervous system } \\
\hline CVA & 25.9 & 58 & 0 \\
\hline Epilepsy & 22.4 & 41 & 7.7 \\
\hline Convulsions & 19.8 & 40 & 17.4 \\
\hline Headache & 14.7 & 35 & 5.9 \\
\hline Meningitis & 8.6 & 24 & 40.0 \\
\hline
\end{tabular}


Table 4 Disposal by diagnostic category

\begin{tabular}{|c|c|c|c|c|c|}
\hline \multirow[t]{2}{*}{ Diagnostic category } & \multicolumn{5}{|l|}{$n(\%)$} \\
\hline & Observed & Admitted & Transferred & LWBS & LAMA \\
\hline Trauma related & $91(12.9)$ & $59(8.4)$ & $21(3.0)$ & $57(8.1 \%)$ & $24(3.4)$ \\
\hline Respiratory tract & $90(31.3)$ & $63(21.9)$ & $1(0.3)$ & $2(0.7 \%)$ & $4(1.4)$ \\
\hline Gastrointestinal tract & $111(37.2)$ & $58(19.5)$ & $4(1.3)$ & $3(1.0 \%)$ & $6(2.0)$ \\
\hline Genital/urethral tract & $33(32.0)$ & $31(30.1)$ & 0 & 0 & $4(3.9)$ \\
\hline Nervous system & $64(59.8)$ & $28(26.2)$ & $7(6.5)$ & $1(0.9 \%)$ & $2(1.9)$ \\
\hline Skin & $24(25.3)$ & $34(35.8)$ & $2(2.1)$ & $4(4.2 \%)$ & $2(2.1)$ \\
\hline Ear nose throat & $7(7.5)$ & $2(2.2)$ & 0 & $1(1.1 \%)$ & $2(2.2)$ \\
\hline Cardiovascular & $52(65.8)$ & $23(29.1)$ & $2(2.5)$ & $2(2.5 \%)$ & $1(1.3)$ \\
\hline Systemic/metabolic & $36(59.0)$ & $18(29.5)$ & $1(1.6)$ & $2(3.3 \%)$ & $1(1.6)$ \\
\hline Musculoskeletal & $8(11.8)$ & $3(4.4)$ & $2(2.9)$ & $2(2.9 \%)$ & $1(1.5)$ \\
\hline Intoxication & $22(46.8)$ & $11(23.4)$ & 0 & $1(2.1 \%)$ & 0 \\
\hline Psychiatric & $10(47.6)$ & $8(38.1)$ & $1(4.8)$ & $1(4.8 \%)$ & $1(4.8)$ \\
\hline Ophthalmic & $1(6.7)$ & 0 & $3(20.0)$ & 0 & 0 \\
\hline Unknown & $16(10.3)$ & $15(9.7)$ & $3(1.9)$ & $70(45.2 \%)$ & $6(3.9)$ \\
\hline
\end{tabular}

This study may be considered a representative sample of the attendees at Paarl-we analyzed a random $14.4 \%$ of all presentations during the study period.

\section{Patient presentation}

A seemingly high proportion of patients $(88.2 \%)$ were selfreferred; Paarl is a secondary level hospital, and one might expect lower self-referral rates (an urban population was found to have a self-referral rate of only $41 \%$ [7]). Of the self-referred cases in our sample, 897 (57.1\%) had triage documented: $81.8 \%$ of these cases were yellow or green. This suggests that the ED deals with a significant primary care load and will have important implications for health policy development. Increased primary care provision and a focused public education campaign on the availability and proper utilization of primary care facilities seem appropriate.

Overall, the majority of patients were triaged yellow, in keeping with other South African data [2, 6, 7]. However, there is clearly a problem with application of the triage tool at Paarl. Focused and ongoing triage training are essential for the successful implementation of the tool.

EMS transport was used by $26.9 \%$ of patients, higher than international studies, which suggest utilization rates between 14 and 20\% [9-11]. Patients who came in with EMS had similar distribution of SATS counts compared to patients who came in using their own transport. However, a large proportion of the population live far from the hospital, and most of them are very poor with limited access to private or public transportation. EMS are free below a certain earnings threshold [5], and therefore its high rate of usage may reflect the socio-economic realities of life in the area.
More trauma cases presented overnight than in the day, as may be expected given standard relationships between trauma and alcohol. South Africa is generally acknowledged to have one of the highest rates of trauma in the world [1]. In total, $36 \%$ of our cases were trauma related, which is a higher rate than other reported South African data $(25-28 \%[6,7])$.

Gunshot wounds are extremely rare in the Paarl area: our sample contained no cases whereas others have reported up to $17.6 \%$ of fatalities in the ED to be due to firearms [12]. We did not analyze the reasons in this study.

There was a high incidence of both gastroenteritis and pneumonia, diseases common in patients with advanced retroviral disease. Human immunodeficiency virus (HIV)/ acquired immunodeficiency syndrome (AIDS) is rife in South Africa, with an estimated $16.6 \%$ affected (adults over 15 years) [13]. We did not analyze whether patients had known tuberculosis (TB) or retroviral disease; we chose to report the presenting complaint only. This may have led to a misrepresentation of the incidence of TB and HIV in our sample.

\section{Future implications}

The initial step in all developmental projects is the needs analysis, i.e. the cross-sectional descriptive study. These studies provide the first "snapshot" data that reveal the initial states of the system and reveal to EM development professionals "where we are now" in terms of the numbers, types and patterns of patients being seen and treatment methods used for any particular system or sub-system. EM systems development includes primary EM development 
(education and patient care systems), secondary EM development (economic and legislative agendas) and tertiary EM development (health policy and public health agendas), and development strategies need to be inclusive of data points, initial states and patterns in all of these areas, and not just in areas concerning clinical care; of course many of these data points are not included in our study. Nevertheless, our cross-sectional descriptive questionnaire provides much of the initial epidemiological needs analysis that can then inform and drive these multiple stages of development, and can be used as a template for similar investigations in the many hundreds of EDs, hospitals and EM systems that are in similar stages of EM development as Paarl Hospital. This study can be used as a template for other similar EDs, hospitals and EM systems that are interested in investigating the states of their own EM systems, and in forming and providing structure for future EM development strategies. While many of these emerging EM systems are likely to be different in terms of levels of training, education, personnel, resources, language and culture, nevertheless the initial data that are provided by a similar questionnaire and similar cross-sectional descriptive data collection tools are essential to any EM development project.

\section{Limitations}

This study did not analyze the day of the week on which patients presented, which would have added helpful information. However, other South African studies reported Mondays and Tuesdays as the busiest days of the week, with the census up to $25 \%$ higher than other days of the week [6, 7].

Our sample included 13 patients who died during their stay in the ED. Unfortunately this number is too small to draw any conclusions about causes of death: as this would add helpful extra information, we undertook a second study, looking only at deaths (over a longer time frame): this will be presented elsewhere.

\section{Conclusion}

We performed a cross-sectional, descriptive study that showed some important demographic, epidemiological and operational features of an ED in rural South Africa. The collection and analysis of these data will no doubt influence the present and future development of EM and acute care systems for Paarl Hospital. These data will help to focus EM development strategies for Paarl Hospital, which will be better driven by the local needs that these data uncover: the heavy preponderance of penetrating trauma in this population indicates that more emphasis needs to be placed on trauma training and trauma systems development for Paarl Hospital, and on the link between pre-hospital services and trauma services that the proper treatment of such trauma patients mandates. Also, the large percentage of self-referred patients with primary care complaints indicates that future EM development must also link with larger development projects including primary care provision. It is these data, derived from a simple epidemiological descriptive tool such as our questionnaire, that highlight those areas in most need of attention and further development.

This study can be used as a template for other similar EDs, hospitals and EM systems that are interested in investigating the states of their own EM systems, and in forming and providing structure for future EM development strategies. Information of this kind is necessary to properly guide the improvement of emergency care and to guide the development of EM in Paarl Hospital, in South Africa, and elsewhere. Ongoing research in this field is required to better understand referral patterns and usage of EDs and other local, regional and national EM epidemiology throughout other areas of South Africa and beyond.

Conflict of Interest None. 


\section{Appendix 1: Questionnaire}

a

Questionnaire Emergency Department

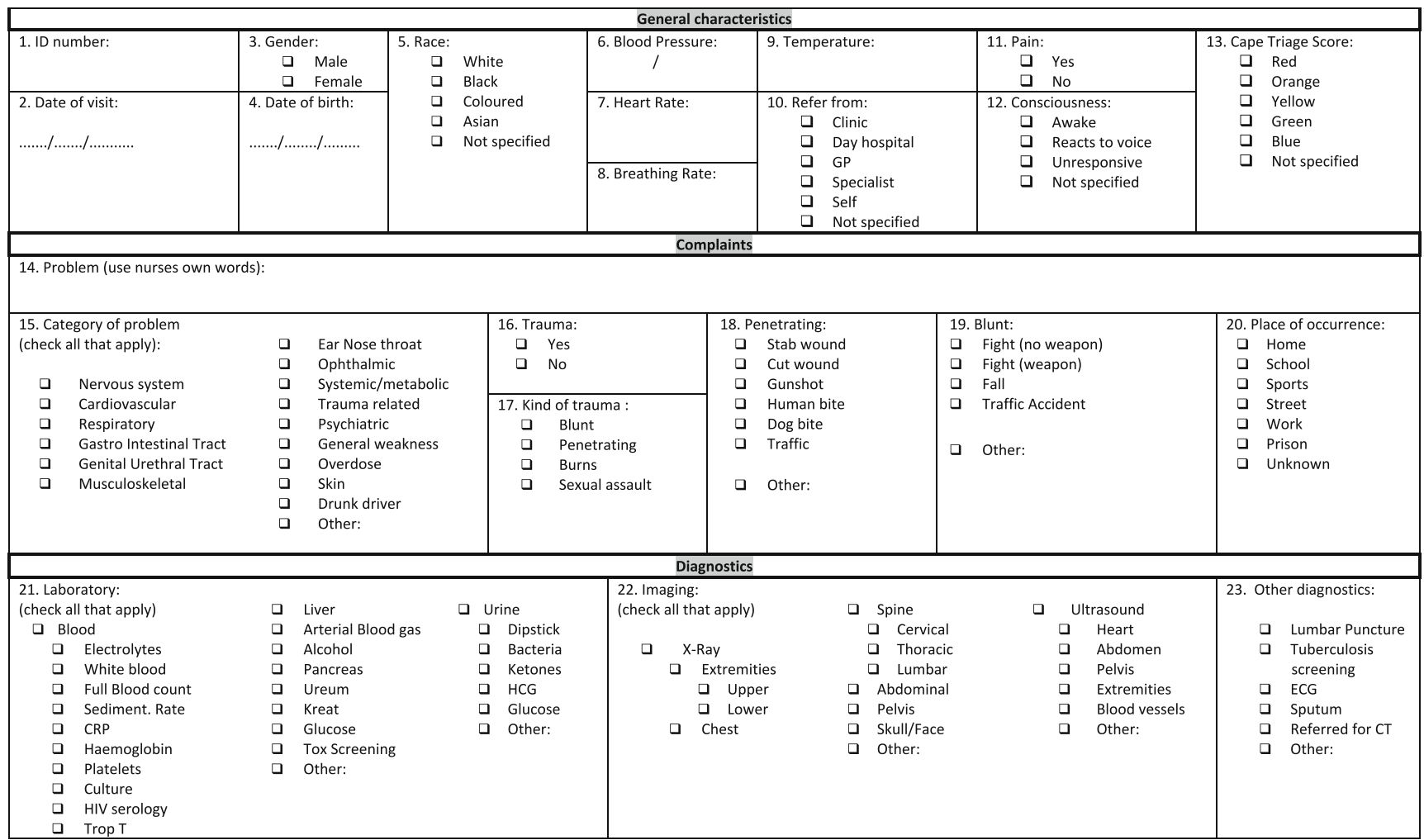

b

Questionnaire Emergency Department

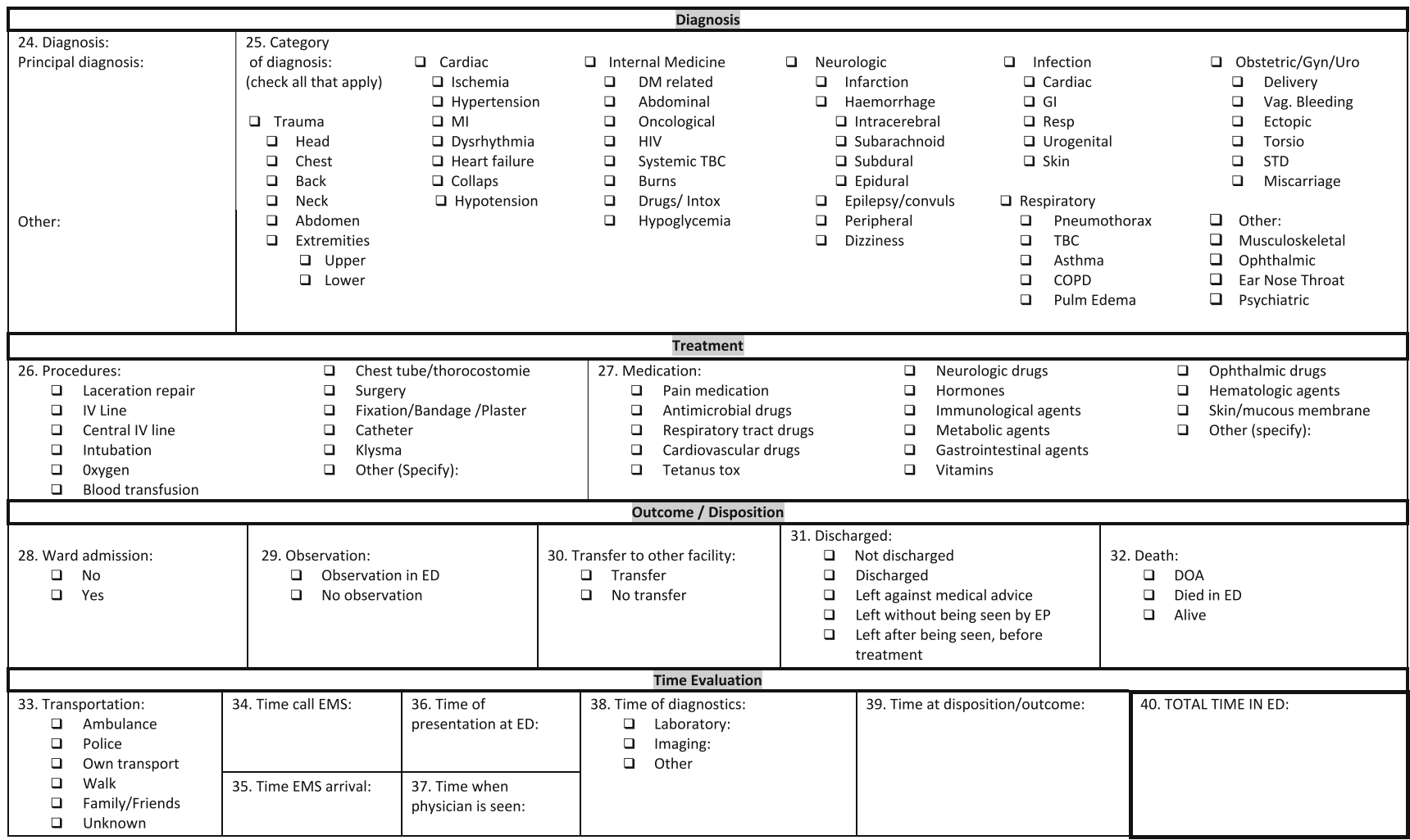




\section{References}

1. Perrott CA (2003) Emergency medicine in South Africa: a personal perspective. J Emerg Med 25(3):325-328

2. Gottschalk SB, Wood D, DeVries S, Wallis LA, Bruijns S, Cape Triage Group (2006) The Cape Triage Score: a new triage system in South Africa. Proposal from the Cape Triage Group. Emerg Med J 23:149-153

3. Dickinson G (1999) African drumbeats: a first conference on emergency medicine. CJEM 1:44-46

4. Balfour C (2006) Emergency medicine-a new era in South African medicine. S Afr Med J 96:47-48

5. MacFarlane C, Loggerenberg C, Kloeck W (2005) International EMS systems: South Africa - past, present, and future. Resuscitation 64:145-158

6. Wallis LA, Twomey M (2007) Workload and casemix in Cape Town emergency departments. S Afr Med J 97:1276-1280

7. Hodkinson PW, Wallis LA (2009) Cross-sectional survey of patients presenting to a South African urban emergency centre. Emerg Med J 26:635-640

8. Website of the South African Triage Group. Available via: www. triagesa.co.za. Accessed 1 Nov 2009

9. de Vries GMJ, Luitse JSK (2001) Emergency medicine in the Netherlands. Ann Emerg Med 38:583-587

10. Burt CW, McCaig LF, Valverde RH (2006) Analysis of ambulance transports and diversions among US emergency departments. Ann Emerg Med 47:317-326

11. Nawar EW, Niska RW (2007). National Hospital Ambulatory Medical Care Survey: 2005 Emergency Department Summary. Advanced Data from vital and health statistics No 386.

12. MRC South Africa (2005). A profile of fatal injuries in South Africa. National injury mortality surveillance system 2005; Section 4: Cape Town Metropolitan Area

13. WHO statistical information system. Available via: http://www. who.int/whosis/en/index.html. Accessed 1 Nov 2009
Rens Hanewinckel is a medical student at the Erasmus University in Rotterdam, The Netherlands. This research was performed in order to accomplish his doctorate degree. Supervision was given by Professor Lee Wallis from the University of Cape Town \& Stellenbosch, South Africa and by Professor Terrence Mulligan from the University of Utrecht, The Netherlands.

Henri P. Jongman is a medical student at the Erasmus University in Rotterdam, The Netherlands. This research was performed in order to accomplish his doctorate degree. Supervision was given by Professor Lee Wallis from the University of Cape Town \& Stellenbosch, South Africa and by Professor Terrence Mulligan from the University of Utrecht, The Netherlands.

Lee A. Wallis is professor of Emergency Medicine at the University of Cape Town and Stellenbosch University, and head of Emergency Medicine for the Provincial Government of the Western Cape. He is president of the Emergency Medicine Society of South Africa, and of the newly formed African Federation for Emergency Medicine.

Terrence M. Mulligan is the Director of the Emergency Medicine Residency and the co-director of the Emergency Department at Universitair Medisch Centrum Utrecht, in Utrecht, The Netherlands since September 2009, where he is working on his $\mathrm{PhD}$ in International Emergency Medicine Development. From 2006-2009, Dr. Mulligan was the Director of the Emergency Department and the Director of the Emergency Medicine Residency at Erasmus Medical Center in Rotterdam, The Netherlands. Dr. Mulligan is also currently a faculty member and a clinical assistant professor at University of Maryland School of Medicine Department of Emergency Medicine since February 2006, where he is active in the International Emergency Medicine program and Fellowship. 